\section{Argentina gives peer review a boost}

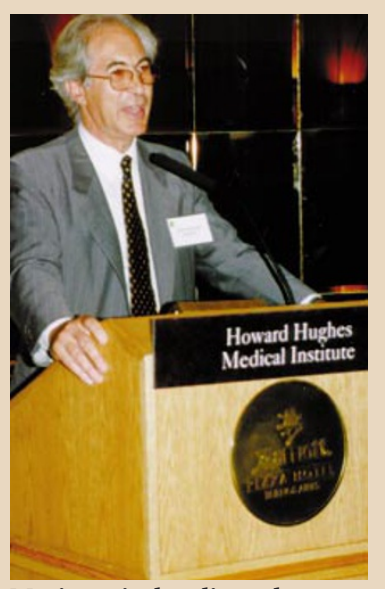

Mariscotti: heading the new agency to promote peer review.

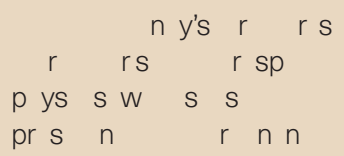

$n$

my

$n$ s. $p p$

$n$ ys rs $r \quad n \quad r n s$

$w r n \quad s \quad s n$

$r n$ n $n$ r $r \quad$ w.

pp ns $r$

$r$ ns $\quad w r$

5000 y $r$ r $r$ y rs

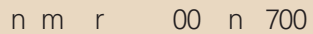

w r sw m

$r$ ns $r \quad x p$

$s p p \quad$ ms $r$

$p \quad p ; y x \quad s \quad r s$

w w n rm y $p$

$y \quad n \quad r s y n$

w r rs r rw $r$.

$r n n n \quad r n m n$

$s n \quad n \quad w$

$n r s$

$r n \quad n n n \quad 50000$

$n \times m r n$

$$
\begin{aligned}
& r n s n s s \\
& r m \quad r n s p n s \\
& n \text { pp } n
\end{aligned}
$$$$
r n m n n \quad n \quad r
$$$$
m r n \quad p m n \quad n
$$$$
\text { ( } w \text { pr ns }
$$$$
\text { n y } x p \quad s
$$$$
\text { pp }
$$$$
\mathrm{pp} \mathrm{n}
$$$$
r \quad w \text { y } q
$$$$
\text { ns }
$$$$
n
$$$$
s \text { pp }
$$$$
s \text { pp ns } r
$$

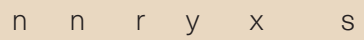

$r$ wsp n n p w r.

$$
\begin{aligned}
& \text { s nss }{ }^{n} m \\
& s
\end{aligned}
$$

collaboration with them (see box, left).

Most of the scientists who have won Hughes funding completed their $\mathrm{PhDs}$ or postdoctoral work in Europe or the United States, and could have prospered there. Yet, according to HHMI officials and the Latin American scientists themselves, the work they presented has some way to go to match that of the Canadian scientists who attended the same meeting.

Many of the scientists see an opportunity ns $r$$$
n n
$$

s pnsw s

$r$ p ns n ss $\begin{array}{llll}r & n & n & n\end{array}$ s s sp $n \quad n$ w $\begin{array}{lllll}n & n & r & n & n\end{array}$ $\begin{array}{llll}n & y & r 1 & 000 .\end{array}$ n $n$ ms

w w srsp ns r

$r n n n n$ rs $s$ $r \quad n \quad p \quad s \quad r n$ $p s n 1 \quad s \quad r s$ w $r s$ $r \quad r n s$ $150 \mathrm{~ns} s \mathrm{~m}$ ys $000 s$ s nss $n$ ny $m \quad n \quad s \quad 00$ $m n \quad r \times r n$ $r$ ns.

ns $\quad s \quad n p y s \quad y$

s ys $n$ rs $s$ s ys ws $\begin{array}{ll}m m & n y \\ w & n\end{array}$

$\begin{array}{llll}n & w & & \\ & n s & s & r\end{array}$

$n \mathrm{p} n \mathrm{n} n \mathrm{~s} s$

$n s m m s p r$

$s$ ys $n$ rs

$r \quad s$

$\begin{array}{rr}n s & r n m n\end{array}$

$r n z \quad n \quad m p$ ys

$m s \quad n r y s \quad n$

$s$ nss $s$ ns rprsn

$$
n \text { w } n y \quad s
$$

$r \quad n s \quad r$

$r$ sm. ss $n$ ys m

$s \mathrm{~ns} s \mathrm{mp} y$ $\mathrm{rnm} n \quad r$

$r s \quad r \quad r \quad r \quad y \quad n \quad s$

$r \quad r r \quad n$

$n \mathrm{p} n \mathrm{n}$

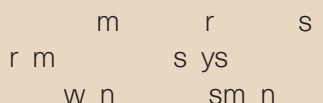

w $n$

w s ss $\quad r m$

$n s$ ys $n s s$

$m$ ns $r$ ns.

$n s \quad$ nys

$n \mathrm{n} n$

s

$$
\begin{aligned}
& s \text { pr zn } \\
& \text { ns } s \text {. }
\end{aligned}
$$

C.M.

to change that. But they acknowledge that the needs of an élite have to be balanced against the need to build a reasonably broad scientific base. Fernando Reinach, a biochemist at the University of São Paulo and part-time head of the life sciences division of FAPESP, the powerful São Paulo state funding agency, says: "In Brazil, if you just support ten groups, you would kill our scientific base. The question is: how do you build the pyramid?"

Science on the continent faces a moment of truth in the face of sweeping economic liberalization, says Reinach. The scientific community, he says, can either come out of this turmoil reinvigorated, as a strong player in the economic progress of these countries or be marginalized as an inefficient machinery incapable of responding to change.

Reinach and Oliva both believe that scientists in Latin America should pay more attention to questions of direct importance to the region. "Scientists need to approach problems that are close to the needs of their own countries," says Oliva. "Some of the good scientists here are too US-orientated," agrees Reinach, who also holds a visiting fellowship at Cornell University in New York. "You don't want to be provincial, but there are unique opportunities here."

He cites the local response to a proposal to sequence the genome of the citrus tree pathogen Xylella fastidiosa (see Nature 389, 654; 1997). "Craig Venter [the prominent US geneticist] thought it was a great idea," he says. "But a lot of people in Brazil weren't sure it was the right thing to do."

Argentina, with its strong scientific tradition and relatively powerful economy, faces problems typical of the continent. Its huge, under-resourced universities pay scant attention to research, and government officials do not feel that the council for science and technology's 150 research institutes are addressing national needs. Its solution is a new research funding agency (see box).

Scientists face many workaday problems too, the most obvious being the delay of urgently needed supplies, such as enzymes, at customs. "Everything to do with buying supplies is extremely complex," says Raúl Padrón, a structural biologist in Caracas and the sole Venezuelan supported by HHMI. "Things will sometimes be damaged because they are not refrigerated properly at customs. They can take months - or a year."

HHMI hopes to gain more support for science through the leverage of its international scholars programme, which gives 20 Canadians and 27 life scientists from Latin America \$50,000 a year for five years to augment their existing funding.

"This obviously won't solve all the problems," says Purnell Choppin, president of HHMI. "But supporting nine or ten highquality scientists in a country does two things. It supports these individuals, and keeps them there; and it tells the government of the country that they have top people that an outside organisation such as HHMI is prepared to support."

The governments may now be ready to rise to that challenge. According to Susana Decibe, the minister of education and culture, Argentina wants to double the proportion of gross domestic product spent on $\mathrm{R} \& \mathrm{D}$, to one per cent. "It's a small figure by the standards of developed countries, but a very ambitious target for us," she says. 